\title{
关于两类图的色多项式
}

目前, 只有为数很少的几类图有色多项 式的计算公式, 而对绝大多数图而言, 计算色 多项式仍是非常不方便的. 本文为两大类图 找到了色多项式的计算公式, 并为寻找更多 的色多项式的计算公式提供了一定的模式. 这两大类图是: 一条长为 $n$ 的路 $P_{n}$ 的补图 $\bar{P}_{n}$ 以及 $n$ 圈的补图 $\bar{C}_{n}$ 。

若图 $G$ 的生成子图 $H$ 的每个分支都是完 全图, 则称 $H$ 为 $G$ 的理想子图, 我们把图 $G$ 的 具有 $k$ 个分支的理想子图的个数记为 $N(G$ ， $k$ ),并用 $f(G, t)$ 表示图 $G$ 的色多项式. 我 们的出发点是

引理 1 若图 $G$ 有 $n$ 个顶点, 那末

$$
\begin{gathered}
f(G, t)=N(\bar{G}, 1)[t]_{1}+N(\bar{G}, 2)[t]_{2}+ \\
\cdots+N(\bar{G}, n)[t]_{n}
\end{gathered}
$$

这里

$$
[t]_{k}=t(t-1)(t-2) \cdots(t-k+1),
$$

$k$ 是自然数。

对于我们所考虑的两类图,首先有

\section{引国 2}

$N\left(P_{n}, k\right)=$

$$
\left\{\begin{array}{c}
\left(\begin{array}{c}
k \\
n+1-k
\end{array}\right) \text { 当 } \frac{n+1}{2} \leqslant k \leqslant n+1 ; \\
0 \quad \text { 当 } 1 \leqslant k<\frac{n+1}{2} .
\end{array}\right.
$$

引理 3

$N\left(C_{n}, k\right)=\left\{\begin{array}{cc}\frac{n}{k}\left(\begin{array}{c}k \\ n-k\end{array}\right) & \text { 当 } \frac{n}{2} \leqslant k \leqslant n ; \\ 0 & \text { 当 } 1 \leqslant k<\frac{n}{2} .\end{array}\right.$

结合引理 1 , 就得到我们的主要结果

\section{定理 1}

$$
f\left(\bar{P}_{n}, t\right)=\sum_{\frac{n+1}{2}<k<n+1}\left(\begin{array}{c}
k \\
n+1-k
\end{array}\right)[t]_{k} .
$$

\section{定理 2}

$f\left(\bar{C}_{n}, t\right)=\sum_{\frac{n}{2}<k<n} \frac{n}{k}\left(\begin{array}{c}k \\ n--k\end{array}\right)[t]_{k},(n \geqslant 4)$.

显然, 利用定理 1 和定理 2 计算 $\bar{P}$ 、和 $\bar{C}_{n}$ 的色多项式是相当方便的.

\section{刘儒英}

（海师范大学数学系，西宁）

\section{楔上的覆盖映照}

在研究微分方程、积分方程的正解时, 需 要讨论楔上或锥.上的映炤是否为同肧. Banach 空间中的集合 $K$ 称为檄, 若 $K$ 是闭凸集， 且 $\forall t \geqslant 0$ 有 $t K \subset K$. 设 $P 、 Q$ 为楔, 称映 照 $f: P \rightarrow Q$ 有线段提升性 质, 若 $\forall x_{0} \in P$, $y_{0}=f\left(x_{0}\right), y_{1} \in Q$, 在 $P$ 中存在道路 $x(t)$, $0 \leqslant t \leqslant 1, x(0)=x_{0}$, 使得

$$
f[x(t)]=(1-t) y_{0}+t y_{1} .
$$

本文证明了
定理 设 $P 、 Q$ 分别是 Banach 空间 $E 、 F$ 中的潔, $f: P \rightarrow Q$ 连续,则 $f$ 是同胚的 充要条件是 $t$ 局部同胚且有线段提升性质.

此定理是著名的 Plastock 定理 (Trans. $A M S, 200(1974) ， 169-183$ ) 在楔上的推 广. 作为推论, 可以把 Hadamard 同胚定理 推广到楔上。

潘兴娬 (山东大学数学系,济南) 